DESY 94-065

$$
\begin{aligned}
& \text { DESY } 94-065 \\
& \text { SW } 9418
\end{aligned}
$$

April 1994

\title{
Spacetime Quantization Induced by Classical Gravity
}

\author{
S. Doplicher \\ Dipart. di Matematica. Linersità di Roma "La Sapienza", Italy \\ Ki. Fredenhagen \\ II. Institut für Theorctische Physik. Iniversität Hamburg \\ J. E. Roberts \\ Dipart. di Matematica. Liniversità di Roma "Tor Vergata", Italy

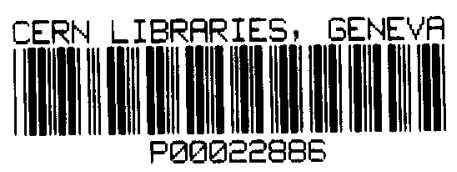

ISS.10 0-118-.98:3:3 



\title{
Spacetime Quantization induced by Classical Gravity
}

\author{
Sergio Doplicher ${ }^{1}$ \\ Dipartimento di Matematica \\ Università di Roma "La Sapienza" \\ I-00185 Roma, Italy \\ Klaus Fredenhagen \\ II Institut für Theoretische Physik \\ Universität Hamburg \\ D-22761 Hamburg, Germany \\ JoIn E. Roberts ${ }^{1}$ \\ Dipartimento di Matematica \\ Università di Rorna "Tor Vergata" \\ I-00133 Roma, Italy
}

\begin{abstract}
We propose spacetime uncertainty relations motivated by Ileisenberg's uncertainty principle and by Einstein's theory of classical gravity. Quantum Spacetime is described by a non-commutative algebra whose commutation relations do imply our uncertainty relations. We comment on the classical limit and on the first steps towards QF' over QST.
\end{abstract}

1. Limitations on the precision of localization in spacetime have appeared in the recent literature as consequences of different approaches to Quantum Gravity $[1,2,3]$ or of some mathematical setup [4]. We propose a converse approach, where operationally motivated, though

\footnotetext{
${ }^{1}$ Research supported by MRST and CNR-GNAPA.
} 
heuristically established, spacetime uncertainty relations are taken as the basis for motivating a novel mathematical model of Quantum Spacetime. The quantum nature of our model manifests itself in commutation relations which are postulated on the basis of the following criteria:

a. They should imply our spacetime uncertainty relations.

b. They should be Poincaré covariant.

c. The commutators should vanish in the large scale limit.

In other words our philosophy is that the quantum deviation of spacetime from its classical structure should be a consequence of the basic principles underlying Quantum Mechanics and General Relativity (point $a$ ). Moreover those deviations should manifest themselves only at the Planck scale, whilst the large scale structure of Quantum Spacetime should be the same as for the usual Minkowski space.

For we are, in a first stage, only interested in idealized states describing a few interacting elementary particles in the absence of very strong external gravitational fields.

The group of global motions of the spacetime should then be the same symmetry group in both cases, including space and time reflections (points $b, c$ ).

As a consequence, ordinary spacetime will appear as translation parameters allowing us to formulate time evolution and wave equations. Furthermore particles are still classified by irreducible representations of the covering group of the restricted Poincare group.

2. Our spacetime uncertainty relations are suggested by Heisenberg's principle and by Einstein's theory of classical gravity. If we perform a very accurate measurement of the spacetime localization of an event, up to uncertainties $\Delta x_{0}, \ldots, \Delta x_{3}$, we must transfer to our testing particles energy of the order $\varepsilon=\frac{\hbar}{a}, a=\min \Delta x_{\mu} \mu=0, \ldots, 3^{2}$. We generate thereby a state which at some time is localized in space with accuracies $\Delta x_{1}, \Delta x_{2}, \Delta x_{3}$ and has an energy momentum tensor $T_{\mu \nu}$ with total ${ }^{2}$ We are interested in the regime where the rest masses of our particles are negligible
with respect to $\varepsilon$. 
energy $\varepsilon$. As an absolute limitation on $\Delta x_{0}, \ldots, \Delta x_{3}$ we adopt the criterion that $T_{\mu \nu}$ should not generate a gravitational field so strong as to trap photons. For otherwise the concentration of energy needed for the localization experiment would have the catastrophic effect of giving rise to black hole formation and thus putting the events under study out of the reach of observation.

It might prove to be a very difficult problem to deduce rigorous bounds for a fairly general class of idealized localized states from this principle. But a heuristic estimate leads to a very reasonable ansatz, namely

$$
\begin{gathered}
\Delta x_{0} \cdot \sum_{j=1}^{3} \Delta x_{j} \gtrsim \lambda_{P}^{2} \\
\sum_{1 \leq j<k \leq 3} \Delta x_{j} \cdot \Delta x_{k} \gtrsim \lambda_{P}^{2} .
\end{gathered}
$$

One is led to these relations by using the linearized approximation to the Einstein equations with a much simplified choice of $T_{\mu \nu}$. Namely, we neglect all other components than the energy density $T_{00}=\rho$ and set, for negative times, $\rho(-t)=\varepsilon \cdot\left(\Delta x_{1}+t\right)^{-1} \cdot\left(\Delta x_{2}+t\right)^{-1} \cdot\left(\Delta x_{3}+t\right)^{-1}$, $t \geq 0$, if $\left|x_{i}\right| \leq \Delta x_{i}+t, i=1,2,3$, and $\rho(-t)=0$ otherwise. In other words we assume uniform spreading towards the past with all speeds not exceeding the speed of light. Here and in what follows we use units $\hbar, c, G$. The associated gravitational potential $\varphi$ at $x_{\mu}=0$ can be written as $\varphi=-\int \frac{\rho(\vec{y},|\vec{y}|)}{|\vec{y}|} \mathrm{d}^{3} y$, and the condition that $|\varphi|$ should be less than the order of unity easily leads to (1), (2).

If $\Delta x_{0}$ is very large we can compare (2) with the stationary solutions of Einstein's equation. If $\Delta x_{i} \sim a, i=1,2,3$, (2) says that $a$ should not be smaller than the Schwarzschild radius for the mass $\frac{1}{a}$. If, say, $\Delta x_{1} \sim \Delta x_{2} \sim r \geq \Delta x_{3} \sim a$, the relation (2) is in agreement with the Kerr solution describing a rotating mass $M \sim \frac{1}{a}$ with radius $r$, for which we must have $M \leq r$ [5]. Further considerations supporting (1), (2) may be found in [6].

3. According to our principles $\mathrm{a}, \mathrm{b}, \mathrm{c}$ we look for commutation relations 
between the selfadjoint coordinate operators $q_{\mu}, \mu=0, \ldots, 3$, acting on a Hilbert space $\mathcal{H}$, which are Poincaré covariant and imply the UR (1), (2). We introduce the antisymmetric tensor $Q_{\mu \nu}$ by

$$
\left[q_{\mu}, q_{\nu}\right]=i Q_{\mu \nu}
$$

and require that the two fundamental invariants which can be formed with $Q_{\mu \nu}$, namely $Q_{\mu \nu} Q^{\mu \nu}$ and $Q_{\mu \nu}\left(* Q^{\mu \nu}\right)=\frac{1}{2} \varepsilon_{\mu \nu \lambda \rho} Q^{\mu \nu} Q^{\lambda \rho}$, should not both be zero.

The simplest further condition on our algebra is that $q_{\mu}$ and $Q_{\lambda \rho}$ commute. As will become clear soon, symmetry considerations lead us to the following Quantum Conditions

$$
\begin{gathered}
Q_{\mu \nu} Q^{\mu \nu}=0, \\
\left(\frac{1}{2} Q_{\mu \nu}(* Q)^{\mu \nu}\right)^{2}=I, \\
{\left[q_{\mu},\left[q_{\lambda}, q_{\nu}\right]\right]=0 .}
\end{gathered}
$$

In generic units, the identity operator on the right hand side of $(4 \mathrm{~b})$ would be multiplied by $\lambda_{P}^{8}$. The square on the l.h.s. is needed to guarantee the symmetry of our QST under time reversal or space reflection. Specific interactions could well break these discrete symmetries, but the basic geometry should be symmetric.

Simplicity motivates (4c), but more significantly it is essential for deducing the UR (1), (2) from the quantum conditions (4), as we now proceed to discuss. The operators $Q_{\mu \nu}$ will be assumed to be (not merely Hermitian but also) selfadjoint and will lie in the centre of our algebra by (4c) (so (4c) is understood to mean that the spectral resolutions of $q_{\mu}$ and $Q_{\lambda \rho}$ commute).

For any state vector $\Phi$ in $\mathcal{H}$, i.e. a vector $\Phi$ with $\|\Phi\|=1$, the uncertainty $\triangle A$ of an observable, described by the selfadjoint operator $A$ acting on $\mathcal{H}$, in the state described by $\Phi$, is given by

$$
(\Delta A)^{2}=\|(A-(\Phi, A \Phi)) \Phi\|^{2}=\left(\Phi, A^{2} \Phi\right)-(\Phi, A \Phi)^{2} .
$$


As is well known, the Schwarz inequality and (3) imply

$$
\left(\Delta q_{\mu}\right)^{2}\left(\Delta q_{\nu}\right)^{2} \geq \frac{1}{4}\left|\left(\Phi, Q_{\mu \nu} \Phi\right)\right|^{2}=\frac{1}{4}\left(\Phi, Q_{\mu \nu}^{2} \Phi\right)-\frac{1}{4}\left(\Delta Q_{\mu \nu}\right)^{2},
$$

where (5) has been used.

Letting $\vec{e}$ and $\vec{m}$ be the "electric" and "magnetic" part of $Q_{\mu \nu}$, respectively, we have $Q_{\mu \nu} Q^{\mu \nu}=\vec{m}^{2}-\vec{e}^{2}, \frac{1}{2} Q_{\mu \nu}\left(* Q^{\mu \nu}\right)=\vec{e} \cdot \vec{m}$, and (4) give

$$
\begin{aligned}
\vec{e}^{2} & =\vec{m}^{2} \\
(\vec{e} \cdot \vec{m})^{2} & =I,
\end{aligned}
$$

whilst (6) gives

$$
\begin{aligned}
\left(\Delta q_{0} \Sigma_{j} \Delta q_{j}\right)^{2} & \geq \frac{1}{4}\left(\Phi, \vec{e}^{2} \Phi\right)-\frac{1}{4} \Sigma_{j}\left(\Delta e_{j}\right)^{2}, \\
\left(\Sigma_{i<k} \Delta q_{j} \cdot \Delta q_{k}\right)^{2} & \geq \frac{1}{4}\left(\Phi, \vec{m}^{2} \Phi\right)-\frac{1}{4} \Sigma_{j}\left(\Delta m_{j}\right)^{2} .
\end{aligned}
$$

We will first show (1), (2) for some special choice of $\Phi$. By (8) we may assume $\vec{e} \cdot \vec{m} \Phi= \pm \Phi$; hence, by the Schwarz inequality, we have

$$
\begin{aligned}
1 & =|(\Phi, \vec{e} \cdot \vec{m} \Phi)| \leq \Sigma_{j}\left|\left(e_{j} \Phi, m_{j} \Phi\right)\right| \leq \\
& \leq \Sigma_{j}\left\|e_{j} \Phi\right\| \cdot\left\|m_{j} \Phi\right\| \leq \\
& \leq\left(\Sigma_{j}\left\|e_{j} \Phi\right\|^{2}\right)^{1 / 2}\left(\Sigma_{j}\left\|m_{j} \Phi\right\|^{2}\right)^{1 / 2}= \\
& =\left(\Phi, \vec{e}^{2} \Phi\right)^{1 / 2} \cdot\left(\Phi, \vec{m}^{2} \Phi\right)^{1 / 2},
\end{aligned}
$$

while by (7) we have $\left(\Phi, \vec{e}^{2} \Phi\right)=\left(\Phi, \vec{m}^{2} \Phi\right)$, so that we also have

$$
\begin{aligned}
\left(\Phi, \vec{e}^{2} \Phi\right) & \geq 1, \\
\left(\Phi, \vec{m}^{2} \Phi\right) & \geq 1 .
\end{aligned}
$$

By virtue of (9) and (10), the U.R. (1), (2) will hold in those states where the $\Delta e_{j}, \Delta m_{k}$ are negligible compared with unity, i.e. where 
$\Phi$ is almost a joint eigenvector of the $Q_{\mu \nu}$ (and hence, as we assumed already, of $\vec{e} \cdot \vec{m}$ ).

Now we take advantage of (4c) to remove these restrictions on $\Phi$. We can choose a partition of the unit $\Sigma_{j} E_{j}=I$, where $E_{j}$ are mutually orthogonal joint spectral projections for the $Q_{\mu \nu}$ 's, associated to spectral sets each having a diameter $\leq \varepsilon$, where $\varepsilon$ is negligible compared to unity. The $E_{j}$ commute with the $q_{\mu}$, and if $\Phi$ is any (unit length) state vector in the domain of $\left[q_{\mu}, q_{\nu}\right]$ s.t. $E_{j} \Phi \neq 0$, the same will apply to $\Phi_{j} \equiv\left(\Phi, E_{j} \Phi\right)^{-\frac{1}{2}} \cdot E_{j} \Phi$. The above argument applies to the uncertainties $\Delta_{j} q_{\mu}$ of $q_{\mu}$ in the state described by $\Phi_{j}$ and, up to $\varepsilon$, (1) and (2) will hold for them.

Now we show that

$$
\Delta q_{\mu} \cdot \Delta q_{\mu} \geq \Sigma_{j}\left(\Phi, E_{j} \Phi\right) \Delta_{j} q_{\nu} \cdot \Delta_{j} q_{\nu}
$$

To see this, set $q_{\mu}^{\prime}=q_{\mu}-\left(\Phi, q_{\mu} \Phi\right)$, and note that in general (cf. (5)) $\|(A-\lambda) \Psi\|^{2}=(\Delta A)^{2}+((\Psi, A \Psi)-\lambda)^{2} \geq\|(A-(\Psi, A \Psi)) \Psi\|^{2}$, so that $\Delta_{j} q_{\mu} \leq\left\|q_{\mu}^{\prime} \Phi_{j}\right\|$. By the definition of $\Phi_{j}$ we can majorize the r.h.s. of (11) by

$$
\begin{aligned}
& \Sigma_{j}\left(\Phi, E_{j} \Phi\right)\left\|q_{\mu}^{\prime} \Phi_{j}\right\| \cdot\left\|q_{\nu}^{\prime} \Phi_{j}\right\|= \\
& \quad=\Sigma_{j}\left\|q_{\mu}^{\prime} E_{j} \Phi\right\| \cdot\left\|q_{\nu}^{\prime} E_{j} \Phi\right\|=\Sigma_{j}\left\|E_{j} q_{\mu}^{\prime} \Phi\right\| \cdot\left\|E_{j} q_{\nu}^{\prime} \Phi\right\| \\
& \quad \leq\left(\Sigma_{j}\left\|E_{j} q_{\mu}^{\prime} \Phi\right\|^{2}\right)^{1 / 2} \cdot\left(\Sigma_{j}\left\|E_{j} q_{\nu}^{\prime} \Phi\right\|^{2}\right)^{1 / 2}=\Delta q_{\mu} \cdot \Delta q_{\nu}
\end{aligned}
$$

where $q_{\mu} E_{j} \Phi=E_{j} q_{\mu} \Phi$ (cf. (4c)) and the Schwarz inequality have been used.

Since (1), (2) hold (up to $\varepsilon$ ) for the $\Phi_{j}$ and $\Sigma_{j}\left(\Phi, E_{j} \Phi\right)=(\Phi, \Phi)=1$, (11) implies that they hold for $\Phi$ too (up to the same arbitrary $\varepsilon$, and hence exactly).

By the Quantum Condition (4), the joint eigenvalues $\left\{\sigma_{\mu \nu}\right\}=(\vec{e}, \vec{m})$ of the commuting selfadjoint operators $Q_{\mu \nu}$ lie in the manifold $\Sigma=$ $\Sigma_{+} \cup \Sigma_{-}$, where

$$
\Sigma_{ \pm}=\left\{\sigma \mid \sigma_{\mu \nu}=-\sigma_{\mu \nu}, \sigma=(\vec{e}, \vec{m}) ; \quad \vec{e}^{2}=\vec{m}^{2}, \quad \vec{e} \cdot \vec{m}= \pm 1\right\}
$$


are connected manifolds where the action of $L_{+}^{\dagger}$ induced by that on 2-tensors is transitive; of course $L$ acts transitively on $\Sigma$ itself.

If we define a Euclidean norm on $\Sigma$ by $\|\sigma\|=\|\vec{e}\|=\|\vec{m}\|=\frac{1}{2} \Sigma_{\mu<\nu} \sigma_{\mu \nu}^{2}$, the unit sphere $\Sigma_{ \pm}^{(1)}$ in $\Sigma_{ \pm}$corresponds to the unit sphere $S^{2}$ in $\mathbb{R}^{3}$ by:

$$
\sigma \in \Sigma_{ \pm}^{(1)} \quad \text { if } \sigma=(\vec{e}, \vec{m}), \quad \vec{e}= \pm \vec{m}
$$

hence iff $\vec{e} \in S^{2}$. Furthermore, $\Sigma_{ \pm}$is topologically equivalent to the tangent bundle $T S^{2}$ of $S^{2}$, or to the left coset space of $S L(2, \mathbb{C})$ modulo diagonal matrices [6].

State vectors $\Phi$ with a very precise localization in spacetime, i.e. such that $\Sigma_{\mu}\left(\Delta q_{\mu}\right)^{2}$ is minimal are concentrated on spectral values of the $Q_{\mu \nu}$ 's lying in $\Sigma^{(1)}$. For, if $\Phi$ is almost an eigenvector of the $Q_{\mu \nu}$ 's with eigenvalues $\sigma \in \Sigma$, we have

$$
\begin{aligned}
& \Sigma_{\mu}\left(\Delta q_{\mu}\right)^{2} \geq \frac{2}{3} \Sigma_{\mu<\nu} \Delta q_{\mu} \Delta q_{\nu} \geq \\
& \geq \frac{1}{3} \Sigma_{\mu<\nu}\left|\left(\Phi, Q_{\mu \nu} \Phi\right)\right| \sim \frac{2}{3}\|\sigma\|,
\end{aligned}
$$

and it is not hard to improve this inequality replacing the r.h.s. by $\sqrt{2}\left(1+\|\sigma\|^{2}\right)^{1 / 2}$; now if $\Phi$ is any unit vector and $Q_{\mu \nu}=\int_{\Sigma} \sigma_{\mu \nu} \mathrm{d} E(\sigma)$ is the spectral resolution of the $Q_{\mu \nu}$ 's, one can prove from the above inequalities that [6]

$$
\Sigma_{\mu}\left(\Delta q_{\mu}\right)^{2} \geq \sqrt{2} \int\left(1+\|\sigma\|^{2}\right)^{1 / 2} \mathrm{~d}(\Phi, E(\sigma) \Phi) .
$$

The minimum of the r.h.s. of (12) is 2 and is reached when the measure $d(\Phi, E(\sigma) \Phi)$ is concentrated on $\Sigma^{(1)}$. As discussed in [6], $\Sigma_{\mu}\left(\Delta q_{\mu}\right)^{2}$ attains this minimum for suitable states over QST. We note here that for the sake of very precise measurements in a given Lorentz frame, $T S^{2}$ effectively shrinks to $S^{2}$, that is $\Sigma$ shrinks to $\Sigma^{(1)}$, the dowoled sphere in $\mathbb{R}^{3}$ of radius $\lambda_{P}^{2}$.

In this context QST is described by the specific algebra of bounded operators discussed in the next section. In the same vein, the usual 
arguments in QM concerning a minimal volume and the number of states in a given volume of phase space carry over to our QST. They tell us that, if we think of the product of the Minkowski space $M_{0}$ and $\Sigma$ as a phase space and ignore the ghost manifold $\Sigma$, the minimal volume in $M_{0}$ is $\left(\sqrt{2 \pi} \lambda_{P}\right)^{4}$.

Furthermore, in any Lorentz frame, there are at most $V /\left(\sqrt{2 \pi} \lambda_{P}\right)^{4}$ independent states over QST approximately localized in a region in Minkowski space with Euclidean volume $V$. Thus the quantum nature of our spacetime automatically entails a discretization of $M_{0}$.

4. The Quantum Conditions discussed in the previous section are the starting point for studying Quantum Spacetime as a novel underlying geometry for QFT. This will be developed elsewhere [6] and here we limit ourselves to outlining the main ideas, the first results and the many problems that arise.

Heisenberg's relations in QM are known to have many singular realizations because $(q, p)$ are unbounded and the same will apply to our relations (4). The regular realizations will be defined by requiring (4) to hold in the more restrictive Weyl form:

$$
e^{i \alpha_{\mu} q^{\mu}} e^{i \beta_{\mu} q^{\mu}}=e^{(i / 2) \alpha_{\mu} Q^{\mu \nu} \beta_{\mu}} e^{i(\alpha+\beta)_{\mu} q^{\mu}},
$$

where $e^{i \alpha_{\mu} q^{\mu}}$ are unitary operators continuous in the real four vector $\alpha$.

Representations fulfilling (13) correspond to (non-degenerate) representations $\pi$ of a $C^{*}$-algebra $\mathcal{E}$ generated by the continuous functions $F$ vanishing at infinity from $\Sigma$ to $L^{1}\left(\mathbb{R}^{4}\right)$. This correspondence is established by the equation

$$
\pi(F)=g(Q) \int f(\alpha) e^{i \alpha_{\mu} q^{\mu}} \mathrm{d}^{4} \alpha
$$

where $F: \sigma \in \Sigma \rightarrow g(\sigma) f$, with $g \in \mathcal{C}_{0}(\Sigma)$ and $f \in L^{1}\left(\mathbb{R}^{4}\right)$.

The ${ }^{*}$ and product operations on $\mathcal{E}$ can be deduced from (14) whilst the $C^{*}$-norm is given by $\sup _{\pi}\|\pi(F)\| . \mathcal{E}$ describes Quantum Spacetime 
and replaces the commutative $C^{*}$-algebra $\mathcal{C}_{0}\left(\mathbb{R}^{4}\right)$ which describes the Minkowski space $\mathbb{R}^{4}$.

The $C^{*}$-algebra $\mathcal{E}$ can be identified with the algebra of all continuous functions vanishing at infinity from $\Sigma$ to the algebra of compact operators over a fixed separable infinite dimensional Hilbert space [6].

In the classical limit $\lambda_{P} \rightarrow 0, \mathcal{E}$ reduces to the commutative algebra $\mathcal{C}_{0}\left(\mathbb{R}^{4} \times \Sigma\right)$, i.e. the QST reduces to $\mathbb{R}^{4} \times\{ \pm 1\} \times T S^{2}$. Thus the ghost manifold $\Sigma$ survives in that limit but, as noted in $\S 3$, only the doubled sphere (of radius $\lambda_{P}^{2}$ ) $\{ \pm 1\} \times S^{2} \sim \Sigma^{(1)}$ is relevant to very precise measurements of localization in a specified Lorentz frame. This doubling of spacetime is reminiscent of Alain Connes' theory of the standard model [7], although the context is different.

One might wonder whether this doubling could be avoided in a variant of our theory, still symmetric under reflections, by setting $Q_{\mu \nu}(* Q)^{\mu \nu}=0$. The arguments of the last section easily show that one of the UR (1), (2) no longer holds in this case. However, changing our Quantum Conditions to $\left(Q_{\mu \nu}(* Q)^{\mu \nu}\right)^{2}=a \cdot I, a>0, Q_{\mu \nu} Q^{\mu \nu}=b \cdot I$ would merely change the numerical values on the r.h.s. of (1) and (2).

The full Poincaré group acts continuously as automorphisms $\tau_{g}$ of $\mathcal{E}$ so that, if $g=(a, \Lambda), \tau_{g}^{-1}$ is induced by the transformation $q_{\mu} \rightarrow$ $(\Lambda q)_{\mu}+a_{\mu}$. The infinitesimal action of translations allows us to define spacetime derivations on $\mathcal{E}$.

The Weyl relations allow us to calculate functions of the quantum position operator $q_{\mu}$ by the von Neumann-Wigner-Moyal formula

$$
\hat{f}(q)=\int f(\alpha) e^{i \alpha_{\mu} q^{\mu}} \mathrm{d}^{4} \alpha
$$

where $f(\alpha)=\frac{1}{(2 \pi)^{2}} \int \hat{f}(x) e^{-i \alpha x} \mathrm{~d}^{4} x$ is an $L^{1}$ function of $\alpha$. These operators form a linear subspace which is not stable under multiplication: $\mathcal{E}$ is spanned, as a normed vector space, by elements of the form (14), i.e.

$$
g(Q) \hat{f}(q), g \in C_{0}(\Sigma), \quad f \in L^{1}\left(\mathbb{R}^{4}\right) .
$$

On these elements it is possible to define spacetime integrals and space 
integrals at a fixed time. More specifically the map

$$
g(Q) f(q) \rightarrow \int_{q_{0}=t} g(Q) f(q) \mathrm{d}^{3} q \equiv g(Q) \int f(\vec{x}, t) \mathrm{d}^{3} x
$$

extends to a positive densely defined linear map of $\mathcal{E}$ into $Z_{0}=\{g(Q)$; $\left.g \in \mathcal{C}_{0}(\Sigma)\right\}$

To get a $\mathbb{C}$-valued analogue of ordinary integration, we may further integrate over $\Sigma^{(1)}$ with the normalized rotation invariant measure, as suggested by our remarks on localization (Section 3). But we have no Lorentz covariant prescription.

This calculus allows us to introduce free fields over $\mathcal{E}$ as operatorvalued functions of the quantum variables $q_{\mu}$. We can furthermore introduce interaction Hamiltonians corresponding to an ordinary Hamiltonian density $\lambda: \phi_{1}(x) \phi_{2}(x) \ldots \phi_{n}(x)$ : (where the summation over spin and internal indices is implicit). Whilst the free Hamiltonian is unchanged by this procedure, one finds that the Hamiltonian density $H_{I}(x)$ gives rise to an interaction Hamiltonian over QST given by

$$
H_{I}(t)=\lambda \int_{\Sigma^{(1)}} \mathrm{d} \sigma \int_{q_{0}=t} \mathrm{~d}^{3} q: \phi_{1}(q) \ldots \phi_{n}(q):=\int_{x_{0}=t} \mathrm{~d}^{3} x H_{e f f}(x),
$$

where $H_{e f f}(x)$ is an effective non-local Hamiltonian density in the free fields over the usual Minkowski space.

For $n=3$, e.g., in generic units, it takes the form

$$
\begin{aligned}
& H_{e f f}(\vec{x}, t)=\frac{1}{\pi^{4}} \int \mathrm{d}^{4} a \mathrm{~d}^{4} b \\
& \quad \lambda: \phi_{1}\left(x+\lambda_{P} a\right) \phi_{2}\left(x+\lambda_{P} b\right) \phi_{3}(x):\left.\right|_{x_{0}=t+\lambda_{p}(b-a)} \cdot \delta(a, b)
\end{aligned}
$$

where the fixed kernel $\delta(a, b)$ is given by

$$
\begin{aligned}
\delta(a, b) & =\int_{\Sigma^{(1)}} \mathrm{d} \sigma e^{-2 i a_{\mu} \sigma^{\mu \nu} b_{\nu}}= \\
& =-\frac{1}{2}\left\{\frac{\sin \gamma_{+}(a, b)}{\gamma_{+}(a, b)}+\frac{\sin \gamma_{-}(a, b)}{\gamma_{-}(a, b)}\right\}, \\
\gamma_{ \pm}(a, b) & =2\left\|a_{0} \vec{b}-b_{0} \vec{a} \pm \vec{a} \times \vec{b}\right\| .
\end{aligned}
$$


The explicit calculations can be found in [6] and yield corrections which are at the lowest order quadratic in $\lambda_{P}$ (unless gravitational forces are explicitly introduced, $\lambda_{P}$ enters our theory only through the commutator $\left[q_{\mu}, q_{\nu}\right]$ which depends on $\lambda_{P}^{2}$ ).

The perturbative expansion of the $S$-matrix based on (16) will of course break Lorentz invariance, a consequence of our definition of space integrals in (16).

One can expect that the ultraviolet divergences are substantially smoothed out by the non-locality introduced by the quantum nature of our spacetime.

5. This note raises a number of questions which will be investigated elsewhere. We mention the more relevant ones.

Locality [8] becomes an asymptotic notion for free fields over QST [6]. Is it stable under (suitable) interactions? Is there a sharp notion of locality?

Scattering Theory should extend naturally to our QST since its large scale behaviour is classical and we have Poincaré symmetry. What are the implications of effective non-locality (cf. (16)) for renormalization theory?

The commutator manifold $\Sigma$ is a mere spectator in the interaction (16). Can the gravitational interaction give a deeper meaning and a dynamical role to $\Sigma$ ?

Gauge Theories over the QST $\mathcal{E}$ ought to be formulated in terms of Alain Connes' non-commutative geometry. Is it possible to formulate Quantum Gravity on $\mathcal{E}$ in a consistent way?

Can curved spacetime be quantized in the same spirit? Black hole formation at a scale larger than the Planck length should of course not be excluded by our quantum conditions. 
Is there a Euclidean version of QFT over QST?

\section{Acknowledgements}

We are pleased to thank D. Buchholz, A. Connes and R. Haag for discussions. S.D. would like to thank C. Sutherland for the warm hospitality extended to him at the University of New South Wales in one stage of this research. Financial support of the Graduiertenkolleg Theoretische Elementarteilchenphysik to S.D. and of the CNR to K.F. is gratefully acknowledged.

\section{References}

[1] D. Amati, M. Ciafaloni, G. Veneziano, Nucl. Physic. B., 347, 551 (1990); D. AMATI, On Spacetime at Small Distances, in Sakharov Memorial Lectures, L.V. KLADYSH and V.YA. FaInBerg eds., Nova Science P. Inc., (1992).

[2] J. Ellis, N.E. Mavromatos, D.V. Nanopoulos, A Liouville String Approach to Microscopic Time in Cosmology, Preprint CERN-TH. 7000/93.

[3] A. Ashtekar, Quantum Gravity: a Mathematical Physics Perspective, Preprint CGPG-93/12-2.

[4] A. KEMPF, Uncertainty Relations in Quantum Mechanics with Quantum Group Symmetry, Preprint DAMT/93-65.

[5] D. Hawking, G.F.R. Ellis, The large scale structure of spacetime, Cambridge U.P., 1973.

[6] S. Doplicher, K. Fredenilagen, J.E. Roberts, The Quantum Structure of Spacetime at the Planck Scale and Quantum Fields, Preprint.

[7] A. Connes, Non Commutative Geometry, Academic Press, to appear.

[8] R. HAAG, Local Quantum Theory, Springer TMP, 1993. 